\title{
Otologic Findings in Patients with Cleft Lip and Palate or Isolate Cleft Palate
}

Lucia K. Carvalhal, MD (presenter); Sady S Costa, MS PhD; Marcus V Collares, PhD; Lisiane S Kruse, MD; Daniela Dall'Inga, $\mathrm{MD}$

Porto Alegre Brazil; Ribeiroao Preto Brazil; Porto Alegre Brazil; Porto Alegre Brazil; Port Alegre Brazil

Objectives: The incidence of middle ear diseases is high in patients with cleft palate. Our objective is to describe otologic findings in 180 patients with cleft lip and palate or isolated cleft palate evaluated and followed at Hospital de Clinicas, Porto Alegre.

Methods: From August 2001 to October 2002, 180 patients were studied at our Otolaryngology and Cleft Palate Clinic. Patients were evaluated with a detailed history and accurate registration of the otoscopic and otomicroscopic findings. Statistical analysis was performed with Student's $t$, Chi-square and Fisher's exact tests.

Results: At initial evaluation, 130 patients (72.2\%) had no otolaryngologic complaints; 109 patients $(70.3 \%)$ had both lip and palate clefts, while $46(29.7 \%)$ had isolated cleft palates. The overall incidence of otoscopic abnormalities was $77.5 \%$. In 80 patients $(46.2 \%)$, there was middle ear effusion, in $11(6.4 \%)$ there was chronic cholesteatomatous otitis media, and in $9(5.2 \%)$ noncholesteatomatous otitis media. In 24 
patients $(13.9 \%)$, moderate to severe retraction of the tympanic membrane was the main finding. There was a linear correlation between patient age and the presence of otolaryngologic findings; middle ear effusions were less frequent with increasing age, whereas the occurrence of chronic conditions such as moderate/ severe retraction and otitis media with or without cholesteatoma were more common among older patients.

Conclusion: Continued otolaryngological evaluation of the patients with cleft palate allows for early intervention and for the preservation of the integrity of the middle ear of these patients. 